Vol. 87, No. 1 - Enero-Febrero 2020. pp 7-12 https://dx.doi.org/10.35366/93261

\title{
Comparación del efecto de la transfusión de concentrado eritrocitario en el contenido arterial de oxígeno de neonatos sin sepsis, con sepsis y choque séptico
}

\author{
Regina Díaz Caneja-Arenas, ${ }^{\ddagger} * *$ José Iglesias-Leboreiro, ${ }^{\S}$ Isabel Bernárdez-Zapata, ${ }^{\S}$ \\ Diana Paulina Orozco-Romero, § Mario Enrique Rendón-Macías ${ }^{\S}, \|$ \\ ${ }^{\ddagger}$ Facultad Mexicana de Medicina de la Universidad La Salle; ${ }^{\S}$ Departamento de Pediatría y Neonatología, Hospital Español de México. \\ II Unidad de Investigación en Análisis y Síntesis de la Evidencia, Instituto Mexicano del Seguro Social, Ciudad de México.
}

\begin{abstract}
RESUMEN
La transfusión de concentrado eritrocitario (CE) en neonatos críticamente enfermos puede justificarse por la intención de incrementar el contenido arterial de oxígeno $\left(\mathrm{CaO}_{2}\right)$, pero es posible que la condición de gravedad modifique su efectividad. Objetivo: Comparar el cambio en el $\mathrm{CaO}_{2}$ postransfusional en neonatos sin sepsis, con sepsis y con choque séptico. Material y métodos: A 110 neonatos, 14 con choque séptico, 38 con sepsis y 58 sin sepsis en su primera transfusión. Se comparó el $\mathrm{CaO}_{2}$ antes y después de la transfusión. $\mathrm{El} \mathrm{CaO}_{2}$ se estableció con la fórmula: $\left.\left(\mathrm{Hb}^{*} 1.34^{*} \mathrm{SatO}_{2}\right)+\left(\mathrm{pO}_{2}{ }^{*} 0.003\right)\right]$ con datos obtenidos por gasometría en sangre arterial. Resultados: Los niveles de hemoglobina postransfusión aumentaron, pero difirió entre grupos, siendo menor en choque séptico: $\sin$ sepsis $13.9 \pm 1.2 \mathrm{~g} / \mathrm{dL}$, sepsis $14.1 \pm 1.6$ y choque séptico $12.5 \pm 2.4$. Lo mismo se observó con el $\mathrm{CaO}_{2} \mathrm{~mL} / \mathrm{dL}, 18.2 \pm 2.02,17.3$ \pm 2.8 y $15.3 \pm 3.6$. Conclusión: Al parecer, la inestabilidad hemodinámica se relaciona con un incremento menor en el $\mathrm{CaO}_{2}$ después de la transfusión de $\mathrm{CE}$. Se requieren más estudios para comprobar estos hallazgos.
\end{abstract}

Palabras clave: Transfusión, paquete globular, contenido arterial de oxígeno, neonato, choque séptico.

\begin{abstract}
Transfusion of erythrocyte concentrate (EC) in critically ill neonates may be justified in the intention of increasing the arterial oxygen content $(\mathrm{CaO} 2)$, it is possible that the serious condition modifies its effectiveness. Objective: To compare the change in post-transfusion $\mathrm{CaO} 2$ in neonates without sepsis, with sepsis and septic shock. Material and methods: 110 neonates in their first transfusion: 14 with septic shock, 38 with sepsis and 58 without sepsis. $\mathrm{CaO} 2$ was compared before and after the transfusion. With data obtained by arterial blood gas, $\mathrm{CaO} 2$ was established with the formula: $\left(\mathrm{Hb}^{*} 1.34\right.$ * SatO2) $\left.+\left(\mathrm{pO} 2{ }^{*} 0.003\right)\right]$. Results: Post-transfusion hemoglobin levels increased, but differed between groups, being lower in septic shock: without sepsis $13.9 \pm 1.2 \mathrm{~g} / \mathrm{dL}$, sepsis $14.1 \pm 1.6$ and septic shock 12.5 \pm 2.4 . The same was observed with $\mathrm{CaO} 2 \mathrm{~mL} / \mathrm{dL}: 18.2 \pm$ $2.02,17.3 \pm 2.8$ and $15.3 \pm 3.6$, respectively. Conclusion: Apparently, hemodynamic instability is related to a minor increase in $\mathrm{CaO} 2$ after EC transfusion. Further studies are required to verify these findings.
\end{abstract}

Keywords: Transfusion, globular package, oxygen arterial content, neonate, septic shock.

*Correspondencia: RDCA, regina29@gmail.com

Conflicto de intereses: Los autores declaran que no tienen.

Citar como: Caneja-Arenas RD, Iglesias-Leboreiro J, Bernárdez-Zapata I, Orozco-Romero DP, Rendón-Macías ME. Comparación del efecto de la transfusión de concentrado eritrocitario en el contenido arterial de oxígeno de neonatos sin sepsis, con sepsis y choque séptico. Rev Mex Pediatr. 2020; 87(1):7-12. doi: 10.35366/93261

[Comparison of the effect of transfusion of red cell concentrate in blood oxygen content of infants without sepsis, with sepsis and septic shock] 


\section{INTRODUCCIÓN}

Transfundir con concentrado eritrocitario (CE) a neonatos críticamente enfermos con o sin anemia ha sido una conducta aceptada para incrementar el contenido arterial de oxígeno $\left(\mathrm{CaO}_{2}\right)$ y con ello mejorar la oxigenación tisular. Por lo anterior, se considera que el $\mathrm{CaO}_{2}$ es un dato confiable y accesible para inferir la oxigenación tisular ${ }^{1-5}$

La trasfusión de CE no está exenta de riesgos, tal como la modificación de la hemodinámica cardiovascular. A diferencia de la incorporación de volúmenes de líquidos, el aporte de CE mantiene un volumen intravascular por mayor tiempo, por lo que esta sobrecarga de volumen puede modificar la estabilidad hemodinámica del paciente. ${ }^{6,7}$

En neonatos el $\mathrm{CaO}_{2}$ se puede modificar por diferentes factores. Uno es la hemoglobina fetal ( $\mathrm{HbF})$; durante situaciones de hipoxia, la concentración de $\mathrm{HbF}$ reduce el riesgo de daño tisular al transportar mayor cantidad de oxígeno, pero con la transfusión de CE con sangre de adultos se disminuye la concentración de $\mathrm{HbF}_{.}^{2,3,8} \mathrm{Otro}$ factor es la sepsis, pero sobre todo el choque séptico que afecta la función capilar con extravasación de líquidos y baja perfusión tisular, lo que altera la distribución de líquidos y sangre. Por lo anterior, la transfusión de CE pudiera no modificar el aporte de oxígeno a los tejidos cuando hay infección grave..$^{9,10}$

Hasta donde sabemos no hay estudios que hayan evaluado el efecto de la transfusión de CE en neonatos con o sin sepsis en el $\mathrm{CaO}_{2}$.

\section{MATERIAL Y MÉTODOS}

Diseño de estudio. Cohorte retrospectiva, observacional, con análisis antes y después.

Participantes. Se incluyeron recién nacidos transfundidos con CE durante su estancia en una Unidad de Cuidados Intensivos Neonatales (UCIN) de un hospital privado en la Ciudad de México durante el periodo de enero de 2011 a diciembre de 2015. Para este estudio, sólo analizamos la primera transfusión de CE (calculada de 10 a $20 \mathrm{~mL} / \mathrm{kg}$ ) de todo paciente que tuviera la medición de los gases sanguíneos arteriales, antes y después de una transfusión. Se excluyeron pacientes a quienes se les realizó exanguinotransfusión y pacientes con cardiopatía cianógena. Cabe señalar que la decisión sobre la transfusión fue individualizada según las necesidades del paciente y a criterio del médico tratante.

Mediciones. De cada paciente se obtuvo edad gestacional, peso, la edad en horas al momento de la transfusión de $\mathrm{CE}$, los niveles de hemoglobina antes y después de la transfusión. Para calcular el $\mathrm{CaO}_{2}$ recabamos los datos gasométricos de presión arterial de oxígeno $\left(\mathrm{PaO}_{2}\right)$ y porcentaje de saturación $\left(\mathrm{SaO}_{2}\right)$, usando la siguiente fórmula:

$$
\mathrm{CaO}_{2}(\mathrm{~mL} / \mathrm{dL})=\left(1.34 *[\mathrm{hb}] * \mathrm{SaO}_{2}\right)+\left(0.003 * \mathrm{PaO}_{2}\right)
$$

Se consideró el valor $\mathrm{CaO}_{2}$ en tres momentos: uno antes de la transfusión $(<24$ horas) y dos después de la transfusión de $\mathrm{CE}$, el primero dentro de las primeras seis horas, y el segundo entre seis y 24 horas posteriores.

Se decidió utilizar un corte a las seis horas debido a que estudios descritos del TRALI (lesión pulmonar aguda producida por transfusión) señalan que en este periodo es cuando se manifiesta inicialmente dicha complicación, mientras que en el otro periodo dentro de las primeras 24 horas se pueden observar otros efectos adversos incluido el TACO (sobrecarga circulatoria por transfusión). ${ }^{11}$ En este punto es conveniente señalar que ningún paciente tuvo alguna complicación secundaria a la transfusión sanguínea.

Además, al momento de la transfusión se tomó en cuenta la condición clínica de los pacientes para agruparlos en tres: 1) sin sepsis, 2) con sepsis, y 3) con choque séptico, es decir, pacientes con inestabilidad hemodinámica por sepsis. Estos tres grupos estuvieron de acuerdo con las definiciones del Consenso Internacional de Sepsis de Pediatría. ${ }^{12-14}$

Análisis estadístico. Los resultados se resumieron por grupos, según su condición clínica al momento de la transfusión de CE. Las variables cualitativas se presentan con frecuencias simples y porcentajes. En el caso de las cuantitativas con promedio y desviación estándar cuando tuvieron distribución normal; en caso contrario, con mediana e intervalos intercuartílicos (Q1, Q3). La comparación entre variables cualitativas se realizó con prueba de $\chi^{2}$. Para la comparación entre grupos de las variables cuantitativas se utilizó análisis de varianza (ANOVA) o prueba de Kruskal-Wallis. Se consideró un nivel de significancia estadística con un valor de $\mathrm{p}<0.05$.

Los diferentes análisis se realizaron con el paquete estadístico SPSS versión $24^{\circledR}$.

Aspectos éticos. El proyecto fue aprobado por el Comité de Investigación y Ética de nuestro hospital, en cumplimiento del Reglamento en Materia de Investigación en Seres Humanos de la Ley General de Salud y en apego a las recomendaciones de la Declaración de Helsinki. Al ser revisión de expedientes, se consideró 


\begin{tabular}{|c|c|c|c|c|c|c|c|}
\hline \multirow[b]{2}{*}{ Datos } & \multicolumn{2}{|c|}{$\begin{array}{l}\text { Sin sepsis } \\
(n=58)\end{array}$} & \multicolumn{2}{|c|}{$\begin{array}{l}\text { Con sepsis } \\
(n=38)\end{array}$} & \multicolumn{2}{|c|}{$\begin{array}{l}\text { Choque séptico } \\
\qquad(n=14)\end{array}$} & \multirow[b]{2}{*}{$p$} \\
\hline & \multicolumn{2}{|c|}{$n(\%)$} & \multicolumn{2}{|c|}{$n(\%)$} & \multicolumn{2}{|c|}{$n(\%)$} & \\
\hline \multicolumn{8}{|l|}{ Sexo } \\
\hline Masculino & \multicolumn{2}{|c|}{$36(62.1)$} & \multicolumn{2}{|c|}{$22(57.7)$} & \multicolumn{2}{|c|}{$8(57.1)$} & $0.89^{*}$ \\
\hline Femenino & \multicolumn{2}{|c|}{$22(37.9)$} & & & \multicolumn{2}{|c|}{$6(42.8)$} & \\
\hline & \multicolumn{2}{|c|}{ Promedio $\pm \mathrm{DE}$} & Prome & DE & \multicolumn{2}{|c|}{ Promedio \pm DE } & \\
\hline SEGC & \multicolumn{2}{|c|}{$31.5 \pm 3$} & & & \multicolumn{2}{|c|}{$31 \pm 3.5$} & $0.37^{\star *}$ \\
\hline Peso en gramos & \multicolumn{2}{|c|}{$1,738 \pm 659$} & \multicolumn{2}{|c|}{$1,550 \pm 672$} & \multicolumn{2}{|c|}{$1,409 \pm 731$} & $0.17^{\star \star}$ \\
\hline Temperatur ${ }^{\circ} \mathrm{C}$ & \multicolumn{2}{|c|}{$37 \pm 0.1$} & \multicolumn{2}{|c|}{$37 \pm 0.1$} & \multicolumn{2}{|c|}{$36.6 \pm 1.3$} & $0.24^{* *}$ \\
\hline $\mathrm{FC}$, latidos/min & \multicolumn{2}{|c|}{$157 \pm 16$} & \multicolumn{2}{|c|}{$160 \pm 14$} & \multicolumn{2}{|c|}{$166 \pm 21$} & $0.16^{\star *}$ \\
\hline $\mathrm{TAM}, \mathrm{mmHg}$ & \multicolumn{2}{|c|}{$43.8 \pm 8.4$} & \multicolumn{2}{|c|}{$41.2 \pm 10.2$} & \multicolumn{2}{|c|}{$39.8 \pm 12.3$} & $0.92^{\star *}$ \\
\hline \multirow[t]{2}{*}{ Hemoglobina g/dL } & \multicolumn{2}{|c|}{$10.2 \pm 1.2$} & \multicolumn{2}{|c|}{$9.8 \pm 1.4$} & \multicolumn{2}{|c|}{$9.8 \pm 3.3$} & $0.13^{\star *}$ \\
\hline & Mediana & Q1-Q3 & Mediana & Q1-Q3 & Mediana & Q1-Q3 & \\
\hline Hematocrito \% & 30.8 & $30.8-33.5$ & 30.3 & 28.3-33.5 & 29.6 & $27.5-31.7$ & $0.48^{\star \star *}$ \\
\hline $\mathrm{SatO}_{2} \%$ & 96.2 & $93.3-98.2$ & 95.8 & $92.9-97.7$ & 96.2 & $92-98$ & $0.67^{\star \star \star}$ \\
\hline $\mathrm{HbF} \%$ & 93 & $89-97$ & 93 & $85.75-97.5$ & 90 & $31.3-97$ & $0.03^{\star \star *}$ \\
\hline
\end{tabular}

$\mathrm{DE}=$ desviación estándar; $\mathrm{SEGC}=$ semanas de edad gestacional corregida; $\mathrm{FC}=$ frecuencia cardiaca; $\mathrm{HF}=$ hemoglobina fetal; $T A M=$ presión arterial media. ${ }^{*} \chi^{2}$ con corrección de Yates. ${ }^{* *}$ ANOVA. ${ }^{* *}$ Kruskal-Wallis.

un estudio sin riesgo, por lo que no requirió de carta de consentimiento informado.

\section{RESULTADOS}

De un total de 1,120 pacientes ingresados en el periodo de estudio, 311 fueron transfundidos, de los cuales sólo se incluyeron 110 neonatos que cumplieron con los criterios de selección. La distribución por grupo fue: 58 $(52.7 \%)$ pacientes sin sepsis, $38(34.5 \%)$ pacientes con sepsis, y $14(12.7 \%)$ pacientes con inestabilidad hemodinámica secundaria a sepsis. Como se observa en la Tabla 1, no encontramos diferencias entre los grupos con respecto a la distribución por sexo, semanas de edad gestacional y el peso al momento de la transfusión.

La edad al momento de la transfusión varió de uno a 19 días de vida, con promedio de siete días de vida. 100 pacientes se transfundieron a una dosis de $20 \mathrm{~mL} / \mathrm{kg} /$ dosis, cinco a $10 \mathrm{~mL} / \mathrm{kg} /$ dosis, y cinco pacientes a $15 \mathrm{~mL} / \mathrm{kg} /$ dosis. Con relación a los valores promedio de hemoglobina pretransfusional tampoco encontramos diferencias entre los grupos. La mayor diferencia se observó en el porcentaje de hemoglobina

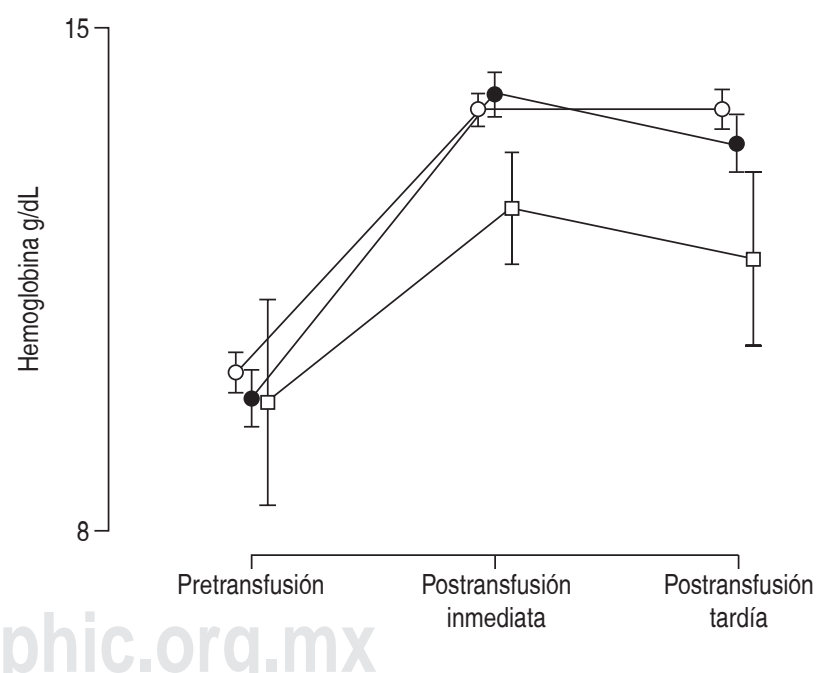

Figura 1: Distribución de niveles de hemoglobina tomadas antes y después de la transfusión de concentrado eritrocitario. Círculos blancos = pacientes sin sepsis; círculos negros = pacientes con sepsis; cuadrados blancos = pacientes con choque séptico. Las marcas muestran los valores promedio por grupo, y las líneas verticales el intervalo de confianza al $95 \%$. 


\begin{tabular}{|c|c|c|c|}
\hline \multirow[b]{2}{*}{ Grupo } & \multicolumn{3}{|c|}{ Contenido arterial de oxígeno (mL/dL) } \\
\hline & $\begin{array}{l}\text { Pretransfusión } \\
\text { Promedio } \pm \text { DE }\end{array}$ & $\begin{array}{l}6 \text { - horas postransfusión } \\
\text { Promedio } \pm \mathrm{DE}\end{array}$ & $\begin{array}{c}6-24 \text { horas postransfusión } \\
\text { Promedio } \pm \mathrm{DE}\end{array}$ \\
\hline Sin sepsis $(n=32)$ & $13.2 \pm 2.1$ & $18.6 \pm 2.09$ & $18.2 \pm 2.02$ \\
\hline Con sepsis $(n=28)$ & $12.6 \pm 2.05$ & $18.4 \pm 2.7$ & $17.3 \pm 2.8$ \\
\hline Con choque séptico $(n=9)$ & $11.9 \pm 3.6$ & $16.8 \pm 2.6$ & $15.3 \pm 3.6$ \\
\hline
\end{tabular}

DE: desviación estándar. ANOVA, Efecto momento $F=150.2_{2 g}, p<0.001$; grupos $F=2 .{ }_{2 g}, p=0.60$ (post-Hoc sin sepsis diferente de sepsis severa-choque séptico); grupo * $F=1.84_{4 \mathrm{gl}} \mathrm{p}=0.12$

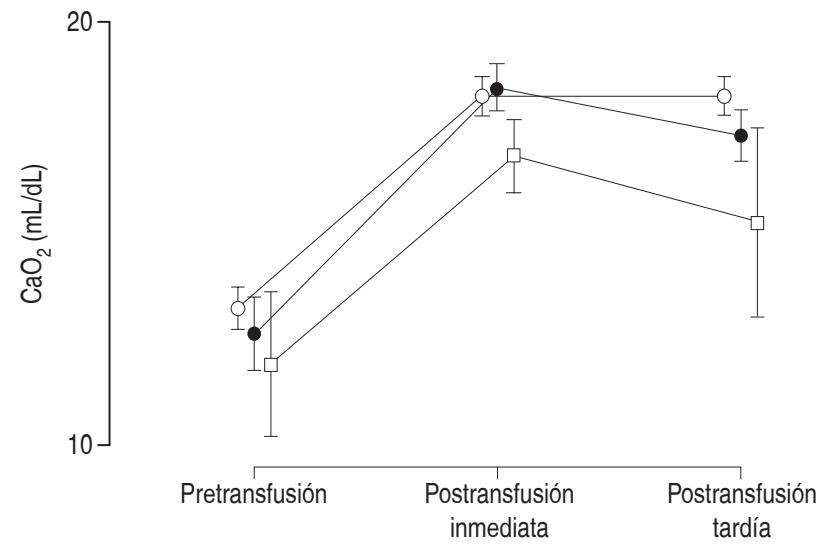

Figura 2: Distribución del contenido arterial de oxígeno (CaO2) antes y después de la transfusión de concentrados eritrocitarios. Círculos blancos = pacientes sin sepsis; círculos negros = pacientes con sepsis; cuadrados blancos = pacientes con choque séptico. Las marcas muestran los valores promedio por grupo, y las líneas verticales el intervalo de confianza al 95\%.

fetal pretransfusional, los pacientes con inestabilidad hemodinámica tuvieron el menor nivel (90\% contra 93\% en los otros dos grupos), lo cual fue estadísticamente significativo $(\mathrm{p}=0.03)$.

Después de la transfusión, en los tres grupos hubo un incremento de los niveles de hemoglobina, y con discreta reducción a las 24 horas. Sin embargo, este incremento fue mayor en los neonatos sin sepsis y en aquéllos con sepsis $(\mathrm{Hb}$ g/dL; sin sepsis pre $=10.2 \pm$ $1.2,<6 \mathrm{~h}=13.9 \pm 1.2,6-24 \mathrm{~h} 13.9 \pm 1.3$; sepsis pre $=$ $9.8 \pm 1.4,<6$ h $14.1 \pm 1.6,6-24$ h $13.4 \pm 1.9)$, en comparación con el grupo con choque séptico (pre $=9.8 \pm$

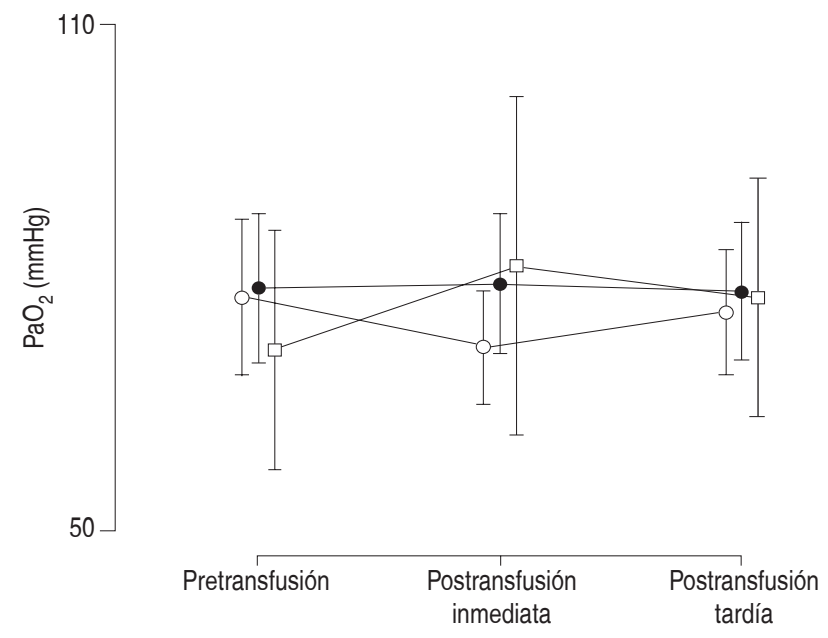

Figura 3: Distribución de los niveles de presión arterial de oxígeno $(\mathrm{PaO} 2)$ tomadas antes y después de la transfusión de concentrado eritrocitario. Círculos blancos = pacientes sin sepsis; círculos negros = pacientes con sepsis; cuadrados blancos = pacientes con choque séptico. Las marcas muestran los valores promedio por grupo, y las líneas verticales el intervalo de confianza al $95 \%$.

$3.4,<6$ h $12.5 \pm 2.4,6-24$ h $11.8 \pm 2.3)$. Esta diferencia fue estadísticamente significativa (ANOVA intragrupo $\mathrm{F}=194,2 \mathrm{gL} \mathrm{p}<0.0001$; intergrupo $\mathrm{F}=5.32 \mathrm{gL}, \mathrm{p}=$ 0.006; Figura 1).

Con respecto al $\mathrm{CaO}_{2}$ (Tabla 2 y Figura 2) después de la transfusión también se observó incremento en todos los grupos, pero fue un poco mayor en los neonatos sin sepsis y con sepsis que en el grupo con choque séptico. Esta diferencia fue estadísticamente significativa; sin embargo, se tiene que considerar que el grupo de 
choque séptico durante los tres momentos mantuvo valores más bajos que los otros dos grupos, y que los niveles de $\mathrm{Hb}$ no aumentaron de la misma forma.

Como se muestra en las Figuras 3 y 4, no hubo diferencia entre los grupos en cuanto a la presión arterial y la saturación de oxígeno.

\section{DISCUSIÓN}

Una disyuntiva en el manejo de neonatos críticamente enfermos, surgida en años recientes, ha sido la decisión de transfundir o no concentrado eritrocitario, ${ }^{13}$ por supuesto en condiciones diferentes a una anemia grave, particularmente durante sepsis. En este estudio, los datos apoyan el beneficio en el incremento del $\mathrm{CaO}_{2}$ después de la transfusión de $\mathrm{CE}$, pero observando que cuando hay choque séptico, es probable que no se llegue a valores normales, es decir, niveles superiores a 15 $\mathrm{mL} / \mathrm{dL}$ de $\mathrm{CaO}_{2}{ }^{15}$

Es posible que los efectos observados del comportamiento de menor $\mathrm{CaO}_{2}$ en neonatos con choque séptico sean consecuencia de varios factores. El primero lo constituyen los niveles de hemoglobina después de la transfusión de $\mathrm{CE}$ que fueron menores en los neonatos sin sepsis. Otra posible explicación pudiera ser porque en sepsis se presenta redistribución de flujos y atrapamiento eritrocitario, hemólisis o sangrados, sobre todo

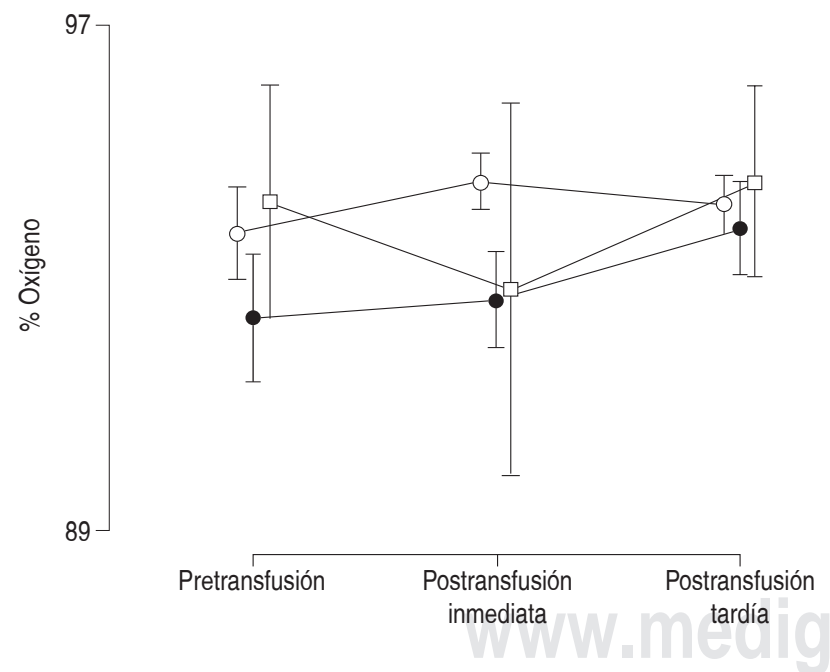

Figura 4: Distribución de la saturación arterial de oxígeno antes y después de la transfusión de concentrados eritrocitarios. Círculos blancos = pacientes sin sepsis; círculos negros = pacientes con sepsis; cuadrados blancos = pacientes con choque séptico. Las marcas muestran los valores promedio por grupo, y las líneas verticales el intervalo de confianza al $95 \%$. cuando hay síndrome de coagulación intravascular diseminada. ${ }^{16-18}$

Por lo anterior, deberemos tomar con reserva que después de la transfusión de $\mathrm{CE}$ en neonatos realmente exista menor incremento en el $\mathrm{CaO}_{2}$ cuando hay choque séptico, lo cual lleva a reflexionar si en estos pacientes se deben transfundir más CE para alcanzar los niveles óptimos de $\mathrm{CaO}_{2}$, pero con riesgo de sobrecarga hídrica, o bien, realizar otras acciones para mejorar la hemodinámica del paciente. Esta situación deberá analizarse en futuros estudios.

Además de lo comentado, otra limitación importante de nuestro estudio es el número de pacientes; como se observa en la Tabla 2, la cantidad de neonatos que tuvieron las tres mediciones de $\mathrm{CaO}_{2}$ fue mucho menor que los pacientes descritos en la Tabla 1. De esta forma, se requieren estudios prospectivos para poder determinar si efectivamente los neonatos con choque séptico tienen un comportamiento diferente del $\mathrm{CaO}_{2}$ tras la transfusión de CE.

\section{CONCLUSIONES}

$\mathrm{Al}$ parecer, el beneficio para mejorar el $\mathrm{CaO}_{2}$ después de la transfusión de concentrado eritrocitario es menor en recién nacidos con choque séptico.

\section{REFERENCIAS}

1. Contreras E. La transfusión de hematíes incrementa la oxigenación tisular y mejora el resultado clínico (pro). Med Intensiva. 2010; 34(7): 467-470.

2. Demetrian M, Illie C, Panaitescu A, et al. Neonates and blood transfusions. Jurnalul Pediatrului. 2015; 18(71-72): 24-28.

3. Roberson RS, Bennett-Guerrero E. Impact of red blood cell transfusion on global and measures of oxygenation. Mt Siani $J$ Med. 2012; 79(1): 66-74.

4. Mendes C, Cardoso-Bourguignon da Silva D, Arduini RG, Troster EJ. Red blood cell transfusion practice in a Pediatric Intensive Care Unit. Einstein (São Paulo). 2011; 9(2): 135-139.

5. Pape A, Stein P, Horn O et al. Clinical evidence of blood transfusion effectiveness. Blood Transfus. 2009; 7: 250-258.

6. Saugel B, Klein M, Hapfelmeir A, Phillip V, Schultheiss C, Meidert AS, et al. Effects of red blood cell transfusion on hemodynamic parameters: a prospective study in intensive care unit patients. Scan J Traum Res Emerg Med. 2013; 21: 21: doi:10.1186/17577241-21-21.

7. Rebel A, Lenz C, Krieter $\mathrm{H}$ et al. Oxygen delivery at high blood viscosity and decreased arterial oxygen content to brains of conscious rats. Am J Physiol Heart Circ Physiol. 2001; 280: H2591-H2597.

8. Shiao SPY, Ou CN, Pierantoni H. The measurement of accurate fetal hemoglobin and related oxygen saturation by the hemoximeter. Clin Chim Acta. 2006; 374(1-2): 75-80.

9. Regueira T, Andresen M. Manipulación del transporte y consumo de oxígeno en la sepsis. Rev Med Chile. 2010; 138: 233-242. 
10. Davis AL, Carcillo JA, Aneja RK, Deymann AJ, Lin JC, Nguyen TC et al. American College of Critical Care Medicine Clinical Practice Parameters for Hemodynamic Support of Pediatric and Neonatal Septic Shock. Crit Care Med. 2017; 45(6): 1061-1093.

11. Roubinian N. TACO and TRALI: biology, risk factors, and prevention strategies. Hematology Am Soc Hematol Educ Program. 2018; 2018(1): 585-594.

12. Collins JA, Rudenski A, Gibson J. Relating oxygen partial pressure, saturation and content: the haemoglobin-oxygen dissociation curve. Breathe. 2015; 11: 194-201.

13. Goldstein B, Giroir B, Randolph A. International pediatric sepsis consensus conference: definitions for sepsis and organ dysfunction in pediatrics. Pediatr Crit Care Med. 2005; 6(1): 2-8.
14. Gutiérrez JA, Theodoru AA. Oxygen delivery and oxygen consumption. In: Pediatric Critical Care. USA: Springer; 2008. pp. 19-38.

15. Román-Vistraín G, Muñoz-Ramírez CM, Márquez-González $H$, Zárate-Castañón $\mathrm{P}$. Valoración hemodinámica durante la guardia. El residente. 2015; 10(2): 69-76.

16. Regueira T, Andresen M. Manipulación del transporte y consumo de oxígeno en la sepsis. Rev Med Chile. 2010; 138: 233-242.

17. Napolitano LM, Kurek S, Luchette FA. Clinical practice guideline: red blood cell transfusion in adult trauma and critical care. Crit Care Med. 2009; 37: 12-23.

18. Chan YL, Han ST, Li CH, Wu CC. Transfusion of red blood cells to patients with sepsis. Int J Mol Sci. 2017; 18: 1946-1949. 